\title{
Instability of conducting polymer sensors in an electronic nose system
}

\author{
E. Schaller ${ }^{1}$, J. O. Bosset ${ }^{*}$ and F. Escher ${ }^{2}$ \\ ${ }^{1}$ Federal Dairy Research Station, CH-3003 Bern, Switzerland \\ ${ }^{2}$ Swiss Federal Institute of Technology (ETH), Institute of Food Science, CH-8092 Zürich, Switzerland
}

\begin{abstract}
A solution of $1 \mathrm{~mL} / \mathrm{L}$ ethanol in water was used to study the repeatability of measurements performed with an electronic nose equipped with 12 conducting polymers based on polypyrrole. The sensor responses exhibited a poor repeatability independently of whether synthetic air or nitrogen was used as carrier gas. The responses varied from one day to the next as well as from one hour to the next. The variations in the baseline resistances, which were also examined, did not help to explain the observed instability. The variations in sensor response could however, be partly explained by a temperature fluctuation of the sensor module. A correction for the temperature changes halved the scatter of the measurements.
\end{abstract}

Keywords. Electronic nose - polypyrrole - conducting polymer - ethanol.

\section{Introduction}

Most studies on electronic nose systems published so far are based on metal oxide semiconductor (MOS), and organic conducting polymer (CP) sensors [1]. These two technologies represent the oldest used in the field of electronic noses, and both rely on changes in resistance due to adsorption of gases. When a voltage is applied across the electrodes, a current passes through the metal oxide or polymer surface. The interaction of volatile compounds with the surface alters the electron flow in the system, and hence, the resistance of the sensor [2]. The mechanisms are however very different. The MOS sensors, working at high temperature, typically 200$600{ }^{\circ} \mathrm{C}$, are based on a combustion principle. The mechanism of $\mathrm{CP}$ sensors, which work at room temperature, is more complex and is not yet completely understood.

$\mathrm{CP}$ sensors comprise a substrate, e.g. fibre-glass or silicon, a pair of gold-plated electrodes, and a conducting organic polymer such as polypyrrole, polyaniline or polythiophene as sensing element. The polymer film is deposited by electrochemical deposition between the two electrodes which have been previously fixed to the substrate [3,4]. As the conducting polymer is grown from a solution, the film contains cation sites balanced by anions from the electrolyte as well as solvent residue [5-7]. The cation sites probably consist of polarons or bipolarons which are small regions of positive charge in the polymer chain providing mobile holes for electron transport. The volatile compounds can interact with at least 3 components within the polymer coating: (i) the polymer itself, (ii) the counterion, or (iii) the solvent [6]. Slater et al. [8] studied the response mechanism of polypyrrole sensors in more detail and showed that the changes in resistance are due to a mixed response involving physical and electronic effects. Certain volatiles have a solvent type action on the polymer causing it to swell, and thus hinder the electron mobility within the coating. The electronic transport process in polypyrrole films can be divided into transport of charge along the polymer chains caused by mobile carriers, such as polarons and bipolarons, and the hopping of charge between the chains. The choice of one process over the other is determined by the conductivity of the film, which in turn is determined by "doping concentrations", the conjugation length of the polymer chain, the chain arrangement, and the position of the counter ions relative to the chains $[9,10]$.

A few studies with electronic noses equipped with $\mathrm{CP}$ sensors concluded that the responses were well reproducible [11-13]. However, most researchers found two main drawbacks for $\mathrm{CP}$ sensor: i) a high sensitivity to moisture, and ii) a rapid drift of their responses over time [3,13-20]. The drift could be partly explained by the oxidation of the polymer coating in contact with volatile compounds and air [21]. Most studies reporting an instability of CP sensors do not give any precise data. They only mention problems of repeatability or a drift of the sensors over time. Few studies $[13,17]$ report in more detail the nature and the amplitude of the changes in the sensor responses and baseline resistances. The instability was noticed over a period of several days, but none of these studies mentioned a instability within one day.

The aim of the present investigation was to measure the repeatability of an electronic nose equipped with $12 \mathrm{CP}$ sensors based on polypyrrole in the course of the day and over a 3-month period. A solution of $1 \mathrm{~mL} / \mathrm{L}$ ethanol in water was

*Correspondence and reprints

Received December 17, 1999; revised February 14, 2000; accepted February 15, 2000. 


\section{Original articles}

used to follow the changes in the sensor response as well as in the baseline resistance.

\section{Experimental part}

An eNose5000 (EEV Limited, Essex, UK) equipped with 12 organic conducting polymer sensors based on polypyrrole was used. The system comprises a CTC Combi PAL autosampler containing 2 trays of 32 vials each.

The headspace is generated statically. The sample to be analysed is placed in an oven at a defined temperature and shaken for a user-defined time. A $5 \mathrm{~mL}$ syringe takes a portion of the headspace and injects it into a $5 \mathrm{~mL}$ Teflon loop where it is trapped by a 6 port valve. After injection of the syringe content, the valve is switched and the volatiles are mixed with a carrier gas at a defined flow rate. The sample is transferred to a control module, which measures the temperature, flow rate and percentage relative humidity of the mixture of volatiles and carrier gas. From this chamber, the gas mixture flows to the sensor module where it is kept for a user-defined time period. The volatile compounds are then purged out through the rear of the instrument. Finally, the sensors are washed with the carrier gas to allow them to return to their baseline resistance values. During the "trap" period, the volatiles interact with the sensors which alters their resistance values. These changes in resistance are converted into a normalised pattern of responses. The data are acquired and displayed by the eNose 5000 software where they can be further analysed.

The baseline is defined as the last raw resistance value before the transfer of volatiles to the sensor array. The sensor responses were defined as the maximum of each curve, and reported as relative value

$$
\frac{\mathrm{dR}}{\mathrm{R}_{\mathrm{b}}}=\frac{\mathrm{R}-\mathrm{R}_{\text {baseline }}}{\mathrm{R}_{\text {baseline }}}
$$

where $\mathrm{R}=$ raw resistance of the sensor and $\mathrm{R}_{\mathrm{b}}=\mathrm{R}_{\text {baseline }}=$ baseline resistance.

Glass vials of $20 \mathrm{~mL}$ were filled with $1 \mathrm{~mL}$ of a solution of $1 \mathrm{~mL} / \mathrm{L}$ ethanol (Merck) in distilled water. The vials were sealed with silicon/ Teflon septa embedded in magnetic caps. Both trays were fully occupied so that each series contained 64 samples. Measurements were carried out each 13 min for 87 days with breaks between measurement series varying from a few minutes to 10 days. A total of 3174 measurements were performed using two different carrier gases: first with synthetic air $\left(\mathrm{N}_{2} / \mathrm{O}_{2}, 80 \% / 20 \%\right.$, technical), and then with nitrogen $\left(\mathrm{N}_{2}\right.$, technical).

The gas flow rate was kept constant at $100 \mathrm{~mL} / \mathrm{min}$. The samples were incubated for $10 \mathrm{~min}$ at $30{ }^{\circ} \mathrm{C}$ and agitated, during incubation, at $500 \mathrm{rpm}$ every $5 \mathrm{~s}$ for $15 \mathrm{~s}$. After the incubation period, $5 \mathrm{~mL}$ of the headspace was injected within $10 \mathrm{~s}$ using a $5 \mathrm{~mL}$ syringe heated at $35^{\circ} \mathrm{C}$ with the following injection parameters: filling speed: 500 , pull-up delay: $500 \mathrm{~ms}$, injection speed: 500, pre-injection delay:
$500 \mathrm{~ms}$, post-injection delay: $500 \mathrm{~ms}$. After a transfer period of $4 \mathrm{~s}$, the volatile compounds were trapped for $2 \mathrm{~min}$ in the sensor chamber for measurement. Before and after each measurement, the sensors were cleaned for $5 \mathrm{~min}$ with the carrier gas, which gave a total cleanup time of $10 \mathrm{~min}$. At the same time, the syringe was flushed with the carrier gas for $85 \mathrm{~s}$ in order to avoid cross-contamination between samples.

\section{Results and discussion}

\section{Sensor responses to the ethanol solution}

Figure 1 represents the maximum responses to the ethanol solution of all $12 \mathrm{CP}$ sensors over the entire 3-month period. The left side shows the measurements performed with synthetic air as carrier gas (1076 measurements), and the right side the measurements carried out with nitrogen (2098 measurements). The $1 \mathrm{~mL} / \mathrm{L}$ aqueous ethanol solution, which is recommended by the manufacturer for calibration, is welldefined, homogeneous and stable compared to other samples such as real food products. However, even after elimination of the outlying responses, the sensor responses obtained were rather unrepeatable. All sensors followed the same trend, i.e. when the response of a given sensor was low or high, all other sensors responded the same way.

The sensors had slightly different responses depending on the carrier gas used. All sensors gave generally higher responses with nitrogen, especially sensor types 458, 297 and 298. However, both carrier gases gave similar response variations, and hence, the same poor repeatability of the measurements. This finding may or may not be in agreement with the results of Bartlett and Ling-Chung [13] who claimed that their laboratory-made CP sensors gave "similar responses" to saturated methanol vapour whether air or oxygen-free nitrogen was used. As no data are given, it is difficult to interpret the exact meaning of "similar responses". If "similar" means the same type of response qualitatively, i.e. curve shape, then our finding agrees with theirs. However, if "similar" means quantitatively, i.e. curve height, then we cannot completely agree with their assertion. As the type of variations observed in our measurements is qualitatively similar for all sensors, it could mean that the sample preparation is responsible for the lack of repeatability. However, it will be shown later that this explanation is not satisfactory.

As all sensors showed the same trend in their response variability, subsequent discussion is based on results obtained with sensor type 458. This arbitrary choice does not influence the discussion or the validity of the conclusions drawn.

Figure 2 presents the relative maximum response values over several days of sensor 458 versus time of measurement. The bottom of the graph corresponds to measurements performed with synthetic air and the top of the graph to measurements carried out with nitrogen. This figure clearly 


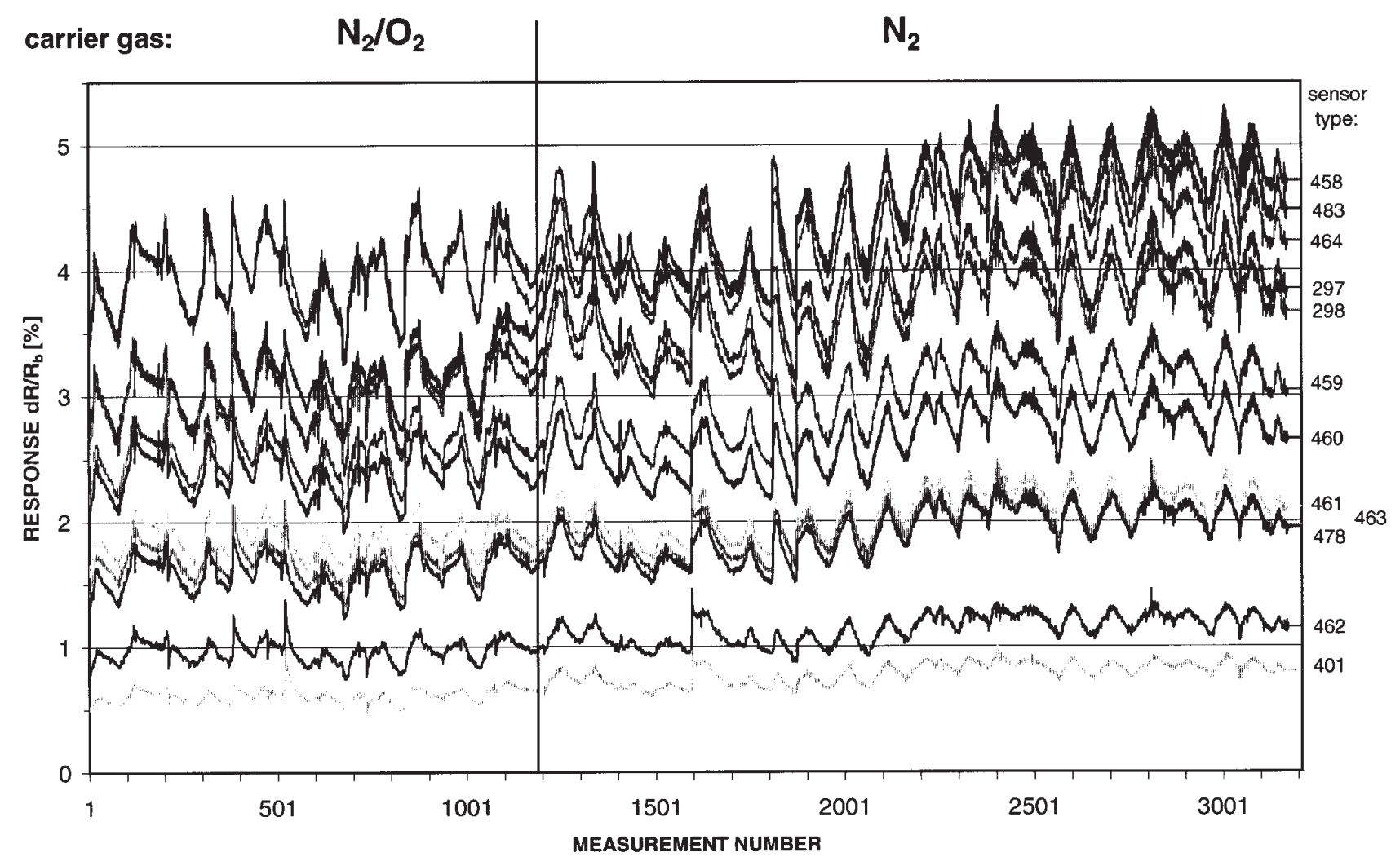

Fig. 1 Maximum response $\left(\mathrm{dR} / \mathrm{R}_{\mathrm{b}}\right)$ of 12 conducting polymer sensors based on polypyrrole to a $1 \mathrm{~mL} / \mathrm{L}$-ethanol solution over a 3-month period. Caption: Each curve represents one sensor.

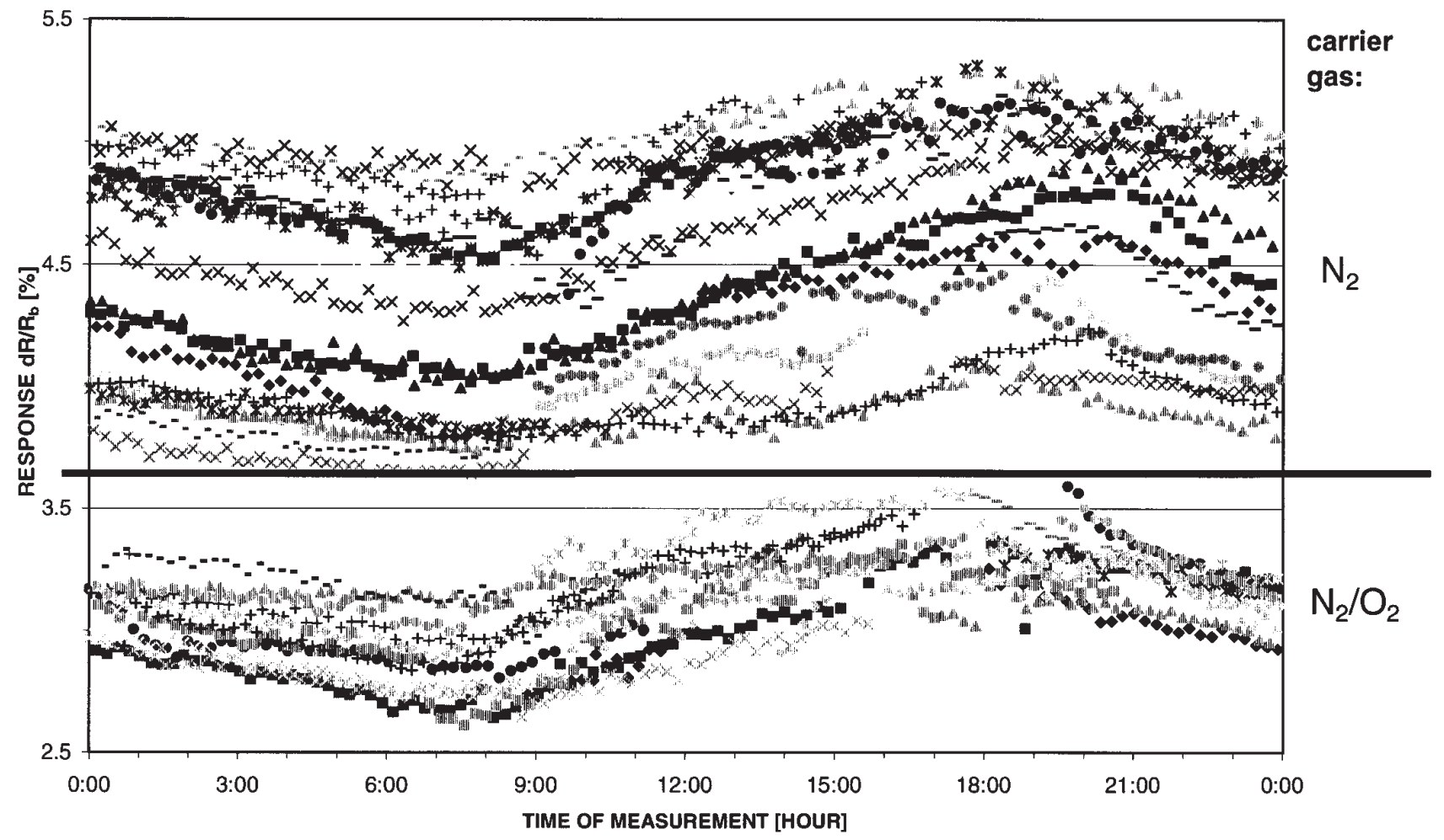

Fig. 2 Maximum response $\left(\mathrm{dR} / \mathrm{R}_{\mathrm{b}}\right)$ of sensor 458 versus time of measurement (hour). Caption: Each symbol represents a day. 


\section{Original articles}

shows that the sensor responses depended on the time of day when the measurements were performed. For both carrier gases, the responses were always the lowest in the morning (around $8 \mathrm{~h}$ ), then rose to reach a maximum in the evening (around $18 \mathrm{~h}$ ), and finally declined again until morning. The same cycle was recorded each day, although the magnitude of the difference between morning and evening measurements can differ significantly.

Table I summarises the sensor fluctuations observed within the same day as well as over the entire period of measurements, i.e. 3 months. The relative fluctuations, $\Delta\left(\mathrm{dR} / \mathrm{R}_{\mathrm{b}}\right)$, represent the difference between the highest and the lowest value of the response $\left(d R / R_{b}\right)$ as related to the average, and were calculated as

$$
\Delta\left(\frac{\mathrm{dR}}{\mathrm{R}_{\mathrm{b}}}\right)=\frac{\left(\mathrm{dR} / \mathrm{R}_{\mathrm{b}}\right)_{\text {max }}-\left(\mathrm{dR} / \mathrm{R}_{\mathrm{b}}\right)_{\min }}{\left(\mathrm{dR} / \mathrm{R}_{\mathrm{b}}\right)_{\text {average }}} * 100
$$

When synthetic air was used as carrier gas, the fluctuations in sensor response within the same day ranged between $44 \%$ (sensor 401) and $7 \%$ (sensor 458, 463 and 298). When nitrogen was used as carrier gas, the fluctuations were between $33 \%$ (sensor 401) and $4 \%$ (sensor 458), and hence were slightly smaller than with synthetic air. The instability observed by us seems however to be much smaller than the one reported by Bartlett and Ling-Chung [13] who noticed a change of 3.6 to $5.0 \mathrm{k} \Omega$ on responses of 3 to $30 \mathrm{k} \Omega$, which gives a variation of 12 to $167 \%$ of the sensor responses.

The hypothesis that the sample preparation alone was responsible for the poor repeatability is shown not to hold because of the fact that the shape of the sensor response in the course of the day was repeatable. However, figure 2 suggests the variation in temperature as another explanation for the trend observed. The measurements were performed during the summer, and there were large temperature differences between morning and evening in the west oriented laboratory. This external temperature significantly affected the temperature of the sensor module. The room temperature was not recorded during the measurements. However, due to the location of the laboratory, the room temperature was always lower in the morning and higher in the evening. The current software does not make possible an automatic recording of the sensor chamber temperature. Therefore, a complete survey of this parameter was not possible. However, it was possible to record the temperature manually as the software always shows the temperature of the module on the screen. This temperature was recorded for several hours and several days.

The sensor chamber is supposed to be kept at a constant temperature of $30{ }^{\circ} \mathrm{C}$, although a variation of $3{ }^{\circ} \mathrm{C}$ was observed during this study. Figure 3 clearly shows that the sensor responses were greatly influenced by an elevation of the sensor chamber temperature. Although data for the highest temperatures are missing, it seems that a small temperature difference between 30.3 and $31.0^{\circ} \mathrm{C}$ has a greater effect on the sensor response than the same difference at higher temperatures. This observation confirms what Yueqiang et al. [9] reported for polypyrrole films. They found that the temperature dependency of the polymer coating was related to the conductivity of the film: the lower its conductivity, the higher the temperature dependency. This observation may explain the greater stability of the sensor response at higher temperatures.

Table I. Fluctuations in the maximum response with synthetic air or nitrogen for each of 12 sensors observed within the same day as well as within the entire period of measurement, i.e. 3 months.

\begin{tabular}{|c|c|c|c|c|c|c|c|c|c|c|c|c|}
\hline \multirow[t]{2}{*}{ Measurements with synthetic air } & \multicolumn{12}{|c|}{ Sensor type } \\
\hline & 458 & 459 & 460 & 461 & 462 & 463 & 464 & 478 & 483 & 297 & 298 & 401 \\
\hline Response fluctuation & 7 & 8 & 8 & 9 & 13 & 7 & 8 & 10 & 8 & 8 & 7 & 10 \\
\hline within one day [\%] & 26 & 28 & 28 & 29 & 42 & 36 & 26 & 34 & 25 & 31 & 31 & 44 \\
\hline Response fluctuation within 3 months [\%] & 31 & 36 & 39 & 42 & 66 & 51 & 34 & 42 & 34 & 44 & 43 & 84 \\
\hline \multirow[t]{2}{*}{ Measurements with nitrogen } & \multicolumn{12}{|c|}{ Sensor type } \\
\hline & 458 & 459 & 460 & 461 & 462 & 463 & 464 & 478 & 483 & 297 & 298 & 401 \\
\hline Response fluctuation & 4 & 6 & 7 & 7 & 8 & 7 & 6 & 7 & 5 & 6 & 6 & 6 \\
\hline within one day [\%] & 21 & 26 & 27 & 30 & 31 & 28 & 25 & 30 & 24 & 27 & 26 & 33 \\
\hline Response fluctuation within 3 months [\%] & 42 & 39 & 41 & 43 & 52 & 46 & 36 & 42 & 35 & 39 & 40 & 51 \\
\hline
\end{tabular}

Caption: The fluctuations, $\Delta\left(\mathrm{dR} / \mathrm{R}_{\mathrm{b}}\right)$, represent the difference between the highest and the lowest value of the response $\left(\mathrm{dR} / \mathrm{R}_{\mathrm{b}}\right)$, and were calculated as follow:

$\Delta\left(\frac{\mathrm{dR}}{\mathrm{R}_{\mathrm{b}}}\right)=\frac{\left(\mathrm{dR} / \mathrm{R}_{\mathrm{b}}\right)_{\text {max }}-\left(\mathrm{dR} / \mathrm{R}_{\mathrm{b}}\right)_{\text {min }}}{\left(\mathrm{dR} / \mathrm{R}_{\mathrm{b}}\right)_{\text {average }}} * 100$, where $\mathrm{R}=$ raw resistance of the sensor, and $\mathrm{dR}=\mathrm{R}-\mathrm{R}_{\mathrm{b}}\left(\mathrm{R}_{\mathrm{b}}=\right.$ baseline resistance $)$. 


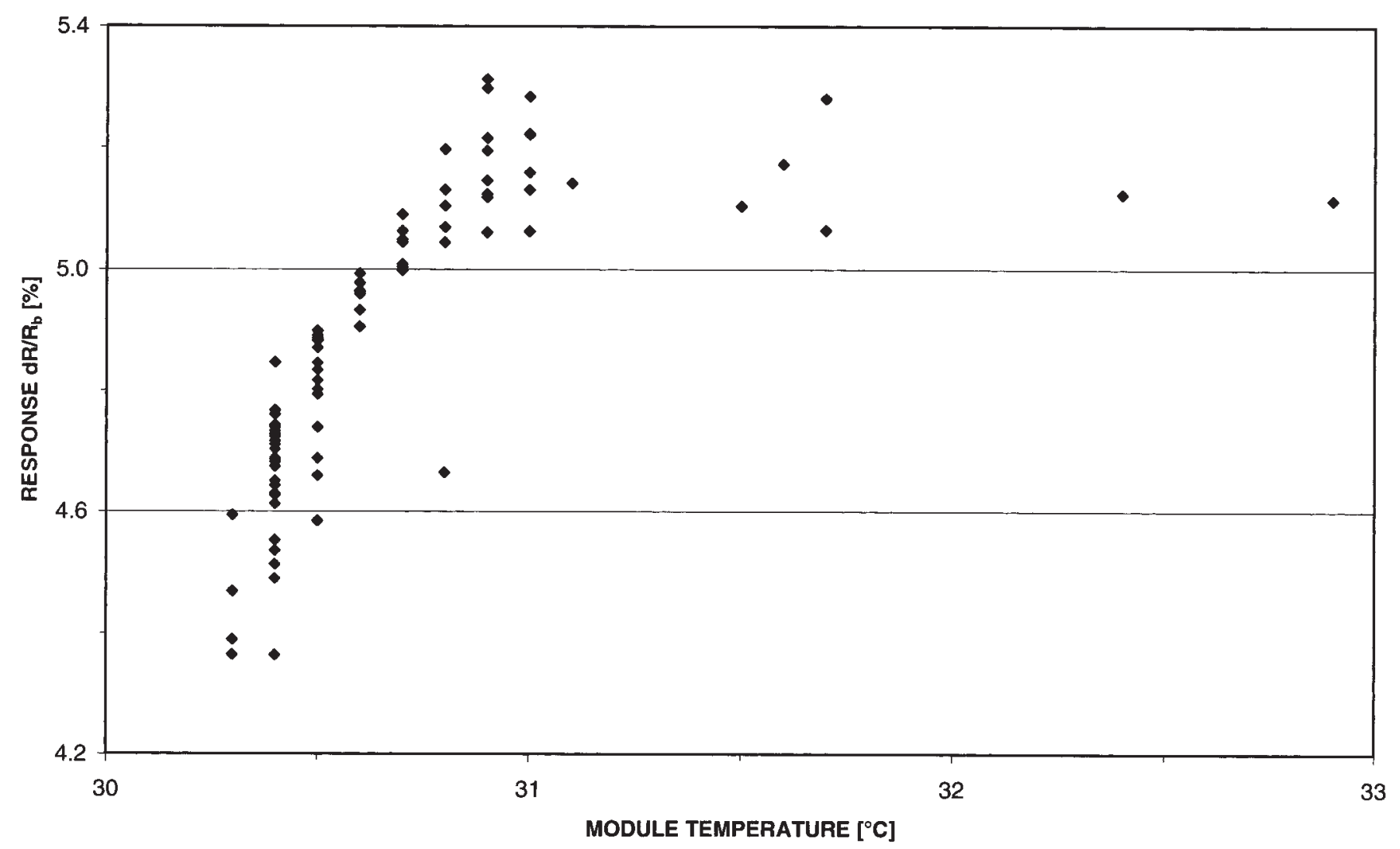

Fig. 3 Maximum response $\left(\mathrm{dR} / \mathrm{R}_{\mathrm{b}}\right)$ of sensor 458 versus temperature $\left({ }^{\circ} \mathrm{C}\right)$ of the sensor module as displayed on the computer screen.

The variation in the module temperature could partly explain the poor repeatability of the results as well as the cyclic shape of the response over 24 hours (Fig. 2). However, measurements performed during days where the room temperature was nearly constant did not show a much better repeatability than measurements carried out when there were large variations in the external temperature between morning and evening. Therefore, this parameter alone can not fully explain the observed instability.

\section{Baseline study}

Pearce et al. [17] suggested that "the change in sensor conductance is a function of the baseline conductance". Therefore, in order to explain the poor repeatability of the sensor responses, the baseline resistance values of the sensors were examined.

Unlike the sensor responses represented in figure 2, figure 4 shows that the baseline values varied more from day to day than within a day; only a small cyclic variation, i.e. maximum of the baseline in the morning and minimum in the evening, could be observed. This statement is confirmed by table II which summarises the baseline fluctuations of each sensor in the course of the day as well as during the entire period of measurement. The fluctuations were calculated in the same way as for the sensor response but by using $R_{\text {baseline }}$ instead of $d R / R_{b}$. Over the entire 3-month period, measurements performed using nitrogen as carrier gas generally showed smaller baseline fluctuations than measurements performed using synthetic air. Whereas the minimum fluctuation within one day for all sensors was much smaller with nitrogen than with synthetic air, the maximum fluctuation was, generally much bigger (except sensor 458). Therefore, the carrier gas used does not seem to influence the baseline stability over a short period of time (one day). However, the baseline values were more stable over a relatively long period of time ( 3 months) when nitrogen was used as carrier gas.

Contrary to other papers [13,17] which report sensor responses 1.5 to 6 times more stable than the baseline resistances, we observed that, independently of the carrier gas used, the baselines were much more stable than the sensor responses. The baseline fluctuation over 3 months was on average only one tenth of the response fluctuation. The maximum baseline fluctuation with nitrogen over one day for sensor 458 was less than one two hundredth of the maximum value of the corresponding sensor response. Thus, although the value of the sensor baseline may be an 


\section{Original articles}

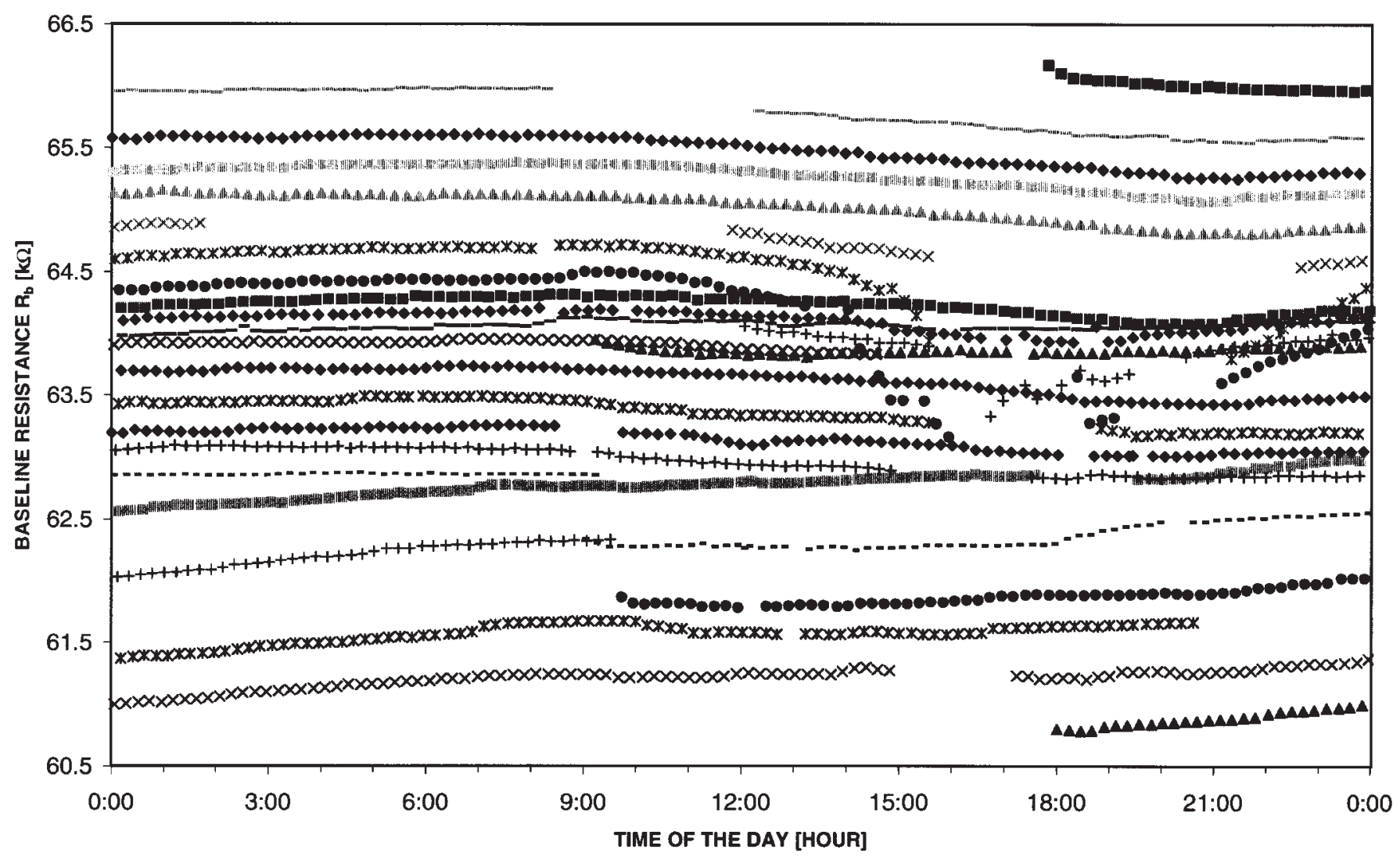

Fig. 4 Baseline resistance $(\mathrm{k} \Omega$ ) of sensor 458 versus time of measurement (hour). Caption: Each symbol represents a day. There was no difference between measurements performed with synthetic air or those carried out with nitrogen as carrier gas.

Table II. Fluctuations in the baseline resistance with synthetic air or nitrogen for each 12 sensors observed within the same day as well as within the entire period of measurements, i.e. 3 months.

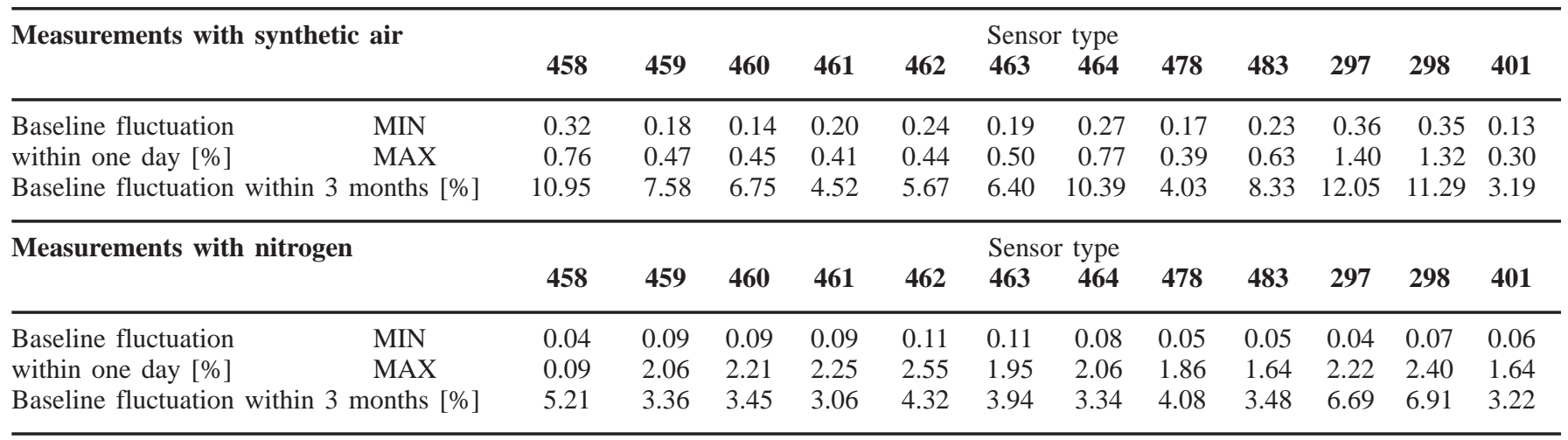

Caption: The fluctuations, $\Delta R_{b}$, represent the difference between the highest and the lowest value of the baseline resistance, $R_{b}$, and were calculated as follow:

$\Delta \mathrm{R}_{\mathrm{b}}=\frac{\left(\mathrm{R}_{\mathrm{b}}\right)_{\text {max }}-\left(\mathrm{R}_{\mathrm{b}}\right)_{\min }}{\left(\mathrm{R}_{\mathrm{b}}\right)_{\text {average }}} * 100$. 
important factor in the repeatability of the sensor response, it is not the only one. In our measurements, the baseline factor played rather a small role in the poor repeatability of the sensor responses, and a direct correlation between the sensor baseline and the sensor response was not apparent. A closer investigation of the baseline variation in the course of the day revealed that the baseline variation showed an opposite trend to the sensor responses, i.e. when the sensor baseline was low, the response to the ethanol solution for the corresponding sensor was high, and vice versa. Figure 5 shows that, although the cycle is clearly repeated, it is difficult to define a mathematical function for the relation between the sensor response and the baseline value.

Figure 6 shows the average baseline resistances for sensor 458 versus the measurement period. When synthetic air was used, the average baseline shows an upward trend. This observation is in accordance with several studies which have reported that $\mathrm{CP}$ sensors based on polypyrrole increase their resistance with time when exposed to air [21,22]. This change in resistance could be explained by an oxidation of the polymer coating. This explanation could also account for the decline of the average baseline values when nitrogen was used. Nitrogen seemed to "regenerate" or reduce the polymer coating to some extend after its exposure to oxidising gases. The baseline increase or decrease was not linear versus time either with synthetic air or with nitrogen and for any of the $12 \mathrm{CP}$ sensors used. When synthetic air was used, the drift was independent of whether the instrument was operating or not, i.e. system completely shut down or withdrawal of the CP module from the system. When nitrogen was used, the sensor baseline resistance increased when the instrument was not on, i.e. the sensors were no longer exposed to nitrogen. This fact supports the hypothesis that the $\mathrm{CP}$ sensors are easily oxidised by air even by a very small concentration [21].

The investigation of the sensor responses revealed a correlation between the sensor response and the temperature of the sensor module (Fig. 3). As expected, a variation of the sensor baseline value was also observed when the chamber temperature increased (Fig. 7). Temperature had opposite effects on the sensor baseline resistance and on the sensor response. Both observations could be related to an increase in the conductivity of the sensor when the temperature increases. Therefore at high temperatures, the sensor conductivity is high, which means a lower baseline resistance value and a higher sensor response. This observation is in agreement with other works [14,22], which report an inverse relation between temperature and baseline resistance of

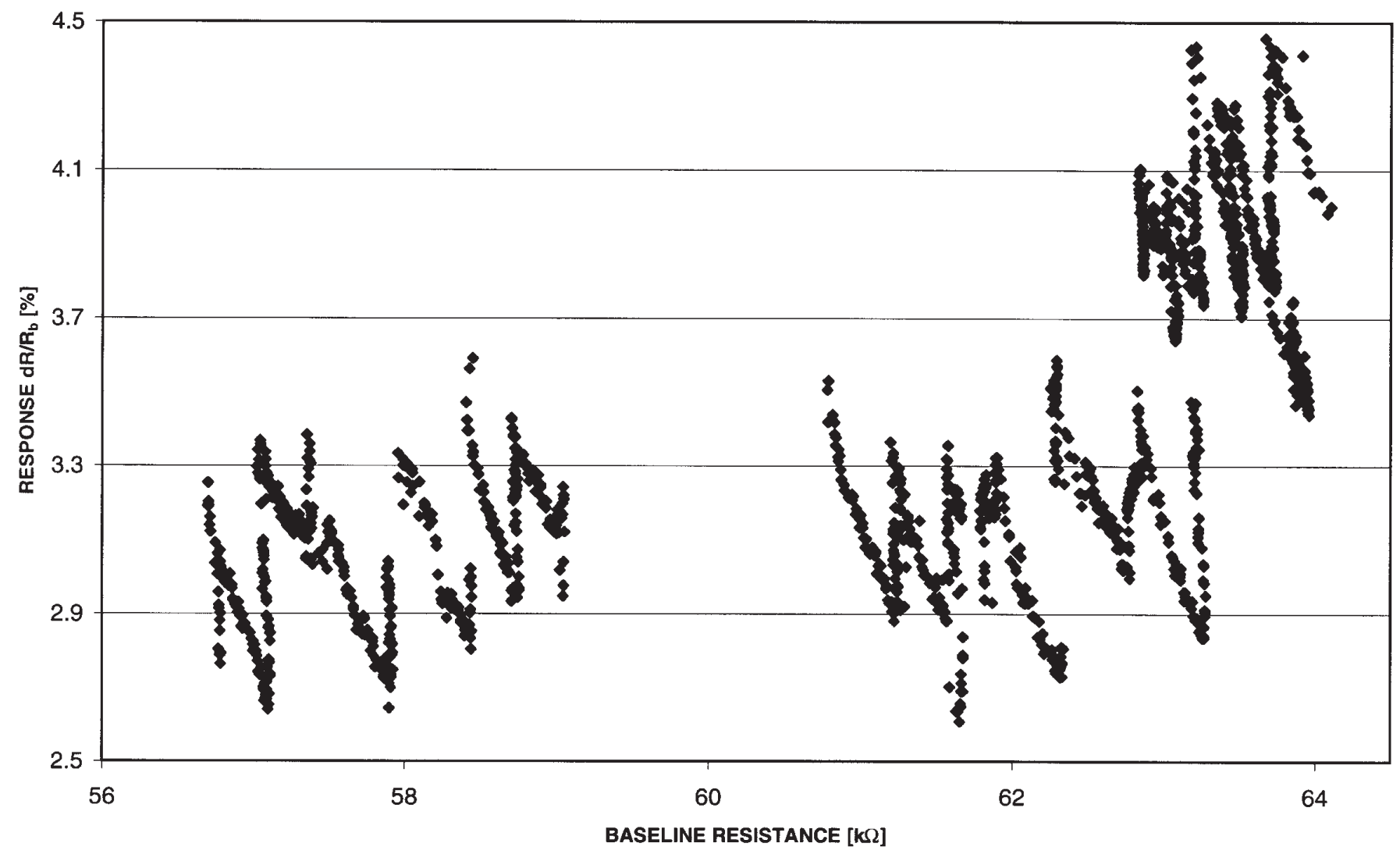

Fig. 5 Maximum response $\left(\mathrm{dR} / \mathrm{R}_{\mathrm{b}}\right)$ of sensor 458 versus baseline resistance $(\mathrm{k} \Omega)$. 


\section{Original articles}

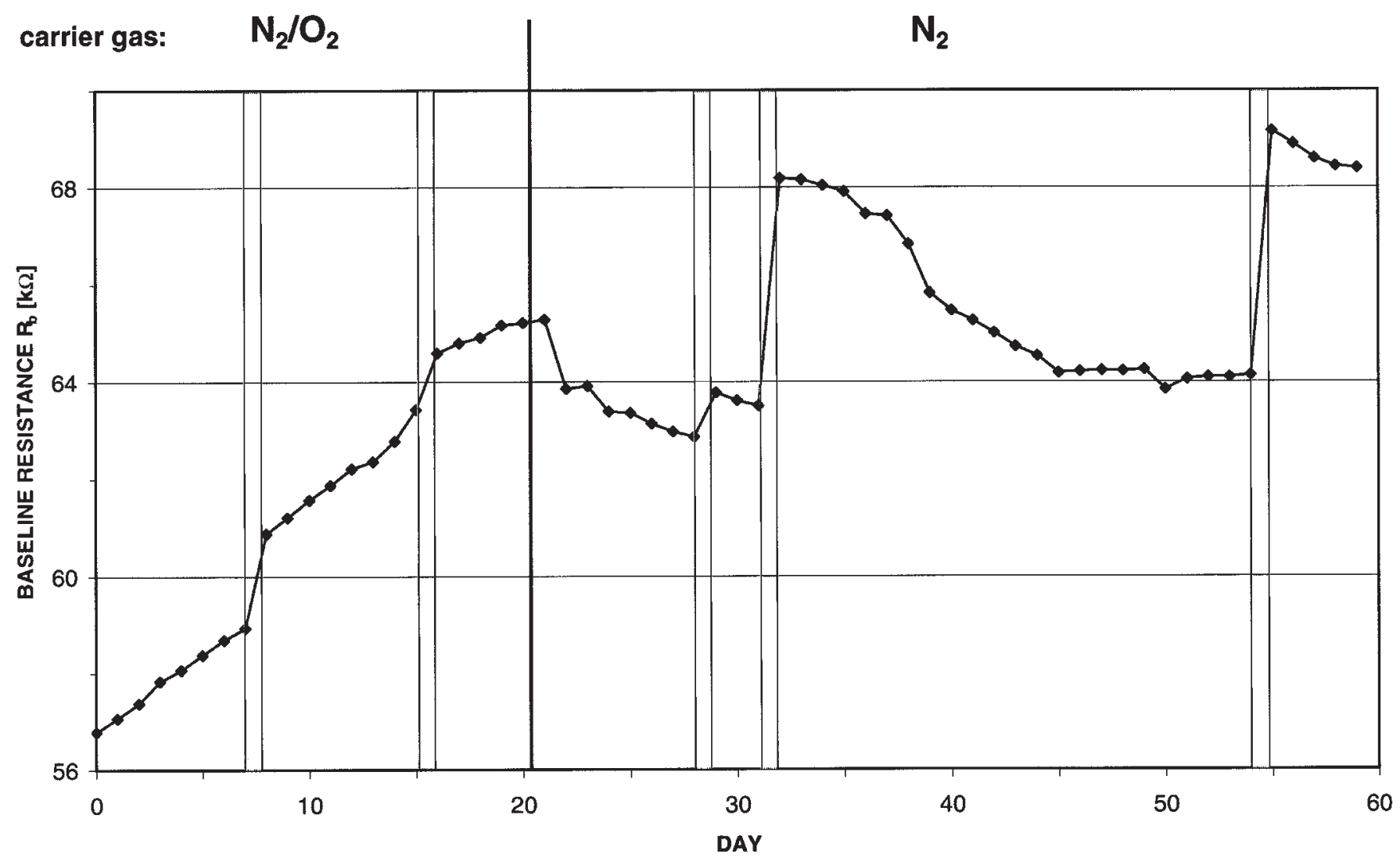

Fig. 6 Daily average baseline resistance $(\mathrm{k} \Omega)$ of sensor 458 versus day of measurement. Caption: The shaded zones correspond to time periods when the sensors were not used.

polymer sensors based on polypyrrole. In the study of Neaves and Hatfield [22], the sensor resistance suffered from a change of $1.25 \Omega /{ }^{\circ} \mathrm{C}$ for an absolute baseline of approximately $100 \Omega$. The sensors from Meijerink [14] were affected of a difference of $5 \Omega /{ }^{\circ} \mathrm{C}$ for an absolute baseline of approximately $500 \Omega$. In our case, the sensors showed a variation of $540 \Omega /{ }^{\circ} \mathrm{C}$ for an absolute baseline of approximately 640 '000 $\Omega$. The ratio of baseline variation to absolute baseline value was 10 times smaller in our case due to a very high absolute resistance.

\section{Attempts at correcting the sensor instability}

A linear parametric drift compensation was applied to the data set corresponding to the sensor responses when the module temperature was registered. This correction was based on the model proposed by Pearce and Gardner [23] where the systematic drift is approximated linearly. In our study, two linear functions were calculated: i) sensor response $\left(d R / R_{b}\right)$ versus time of day, and ii) sensor response $\left(d R / R_{b}\right)$ versus temperature of the sensor module. The slopes of the linear functions obtained were then used to correct the sensor responses by using the equation

$$
\left(\mathrm{dR} / \mathrm{R}_{\mathrm{b}}\right)_{\text {corr }}=\left(\mathrm{dR} / \mathrm{R}_{\mathrm{b}}\right)_{\text {uncorr }}-\mathrm{a} * \mathrm{t},
$$

where $\left(\mathrm{dR} / \mathrm{R}_{\mathrm{b}}\right)_{\text {corr }}=$ corrected sensor response, $\left(\mathrm{dR} / \mathrm{R}_{\mathrm{b}}\right)_{\mathrm{uncorr}}=$ uncorrected sensor response, $\mathrm{a}=$ slope of the linear function, and $\mathrm{t}=$ time or temperature of measurement.

As the sensor responses taken into account for the time of day correction were collected between 7 am and $8 \mathrm{pm}$, the instability in the course of the day could be considered as approximately linear. The slope for correcting the systematic instability caused by temperature changes was calculated between 30.3 and $31.0^{\circ} \mathrm{C}$, as linear approximation cannot be done for the entire range, and only too limited a number of points were available for higher temperatures.

Figure 8 a) shows measurements without any correction, i.e. as they are registered by the electronic nose software. On this graph, one could see a dispersion of the data set of $1 \%$, i.e. the sensor responses range from 4.3 to $5.3 \%$. Figure $8 \mathrm{~b}$ ) displays the same data set with the time of day as correctiong factor, i.e. "a" in equation (1) was replaced by the slope obtained on figure 2 when only measurements between 7 am and $8 \mathrm{pm}$ are taken into account, and "t" was replaced by the corresponding time of the day. Figure $8 \mathrm{c}$ ) shows always the same data set but this time the temperature of the sensor module was used as correcting factor, i.e. "a" in equation (1) was replaced by the slope obtained on 


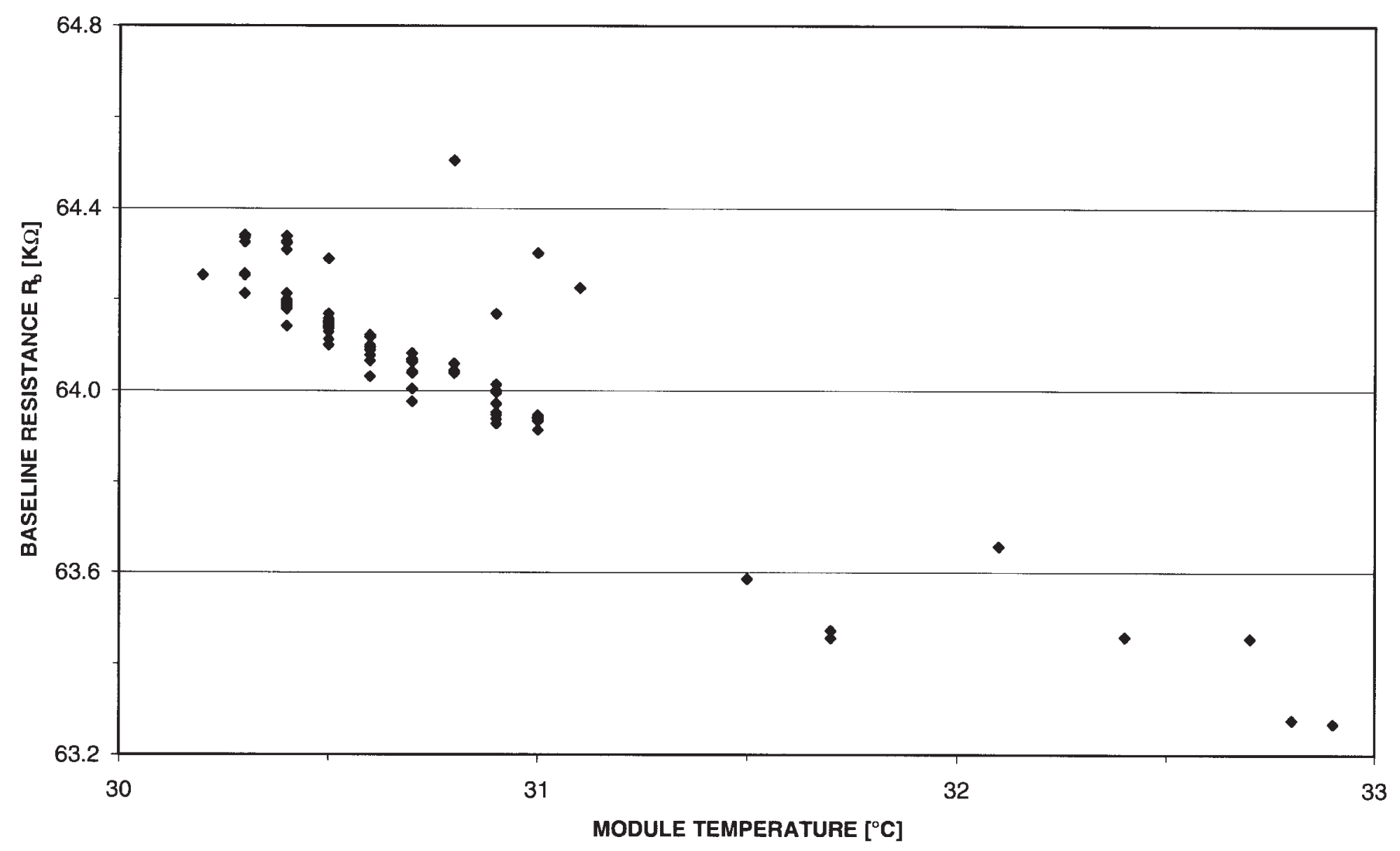

Fig. 7 Baseline resistance $(\mathrm{k} \Omega)$ of sensor 458 versus temperature $\left({ }^{\circ} \mathrm{C}\right)$ of the sensor module as displayed on the computer screen.

figure 3 by considering temperatures between 30.3 and $31.0^{\circ} \mathrm{C}$, and " $\mathrm{t}$ " stood for the temperature of the sensor module. The compensation applied using temperature gave much better results than the one applied using the time of day instability. The mathematical correction for the temperature variation was able to halve the scattering of the measurements, going from $1 \%$ to $0.5 \%$ dispersion. The correction applied using the time of day, on the other hand, did not improve the data dispersion. The significant reduction in the sensor response dispersion with the temperature compensation confirms the hypothesis that temperature variation of the system is a major cause of the instability of the sensor response. At least half of the response instability can be explained by a temperature variation. The other half could be due to noise in the polymer itself [14].

\section{Conclusions}

The results obtained show that electronic noses equipped with conducting polymer sensors may not be as simple to use as they may look first. Even with simple, homogeneous and stable samples such as $1 \mathrm{~mL} / \mathrm{L}$ ethanol in water, only poor repeatability was observed. Continuous series of measurements over several days showed that the sensor responses varied not only from day to day, as already reported in many other studies, but also from hour to hour. Lack of repeatability with CP sensors is often explained by a difference in the water content in the samples. Since our samples all had the same water content, the poor repeatability could not be related to this parameter, and other causes were investigated. Unlike in other studies $[13,17]$, the baseline values only affected to a small extent the repeatability of the sensor response, and could be considered as nearly negligible. The temperature of the sensor chamber, and therefore of the sensors themselves, was found to have a much greater influence on the sensor responses. However, these two parameters did not explain the whole instability observed during this study, and further still unknown factors should be identified and investigated to completely understand the poor repeatability of the CP sensors. No simple correlation could be found between the sensor responses and the corresponding baseline or temperature values. Therefore, an easy correction of the sensor response based on these two parameters was not possible, and any calibration would be very difficult to establish due to the huge and rapid variation of the sensor responses.

Therefore, the eNose 5000 equipped with CP sensors was shown to be inappropriate for most applications. Potential applications with this technology would require a precise temperature control of the sensors, and would be only 


\section{Original articles}

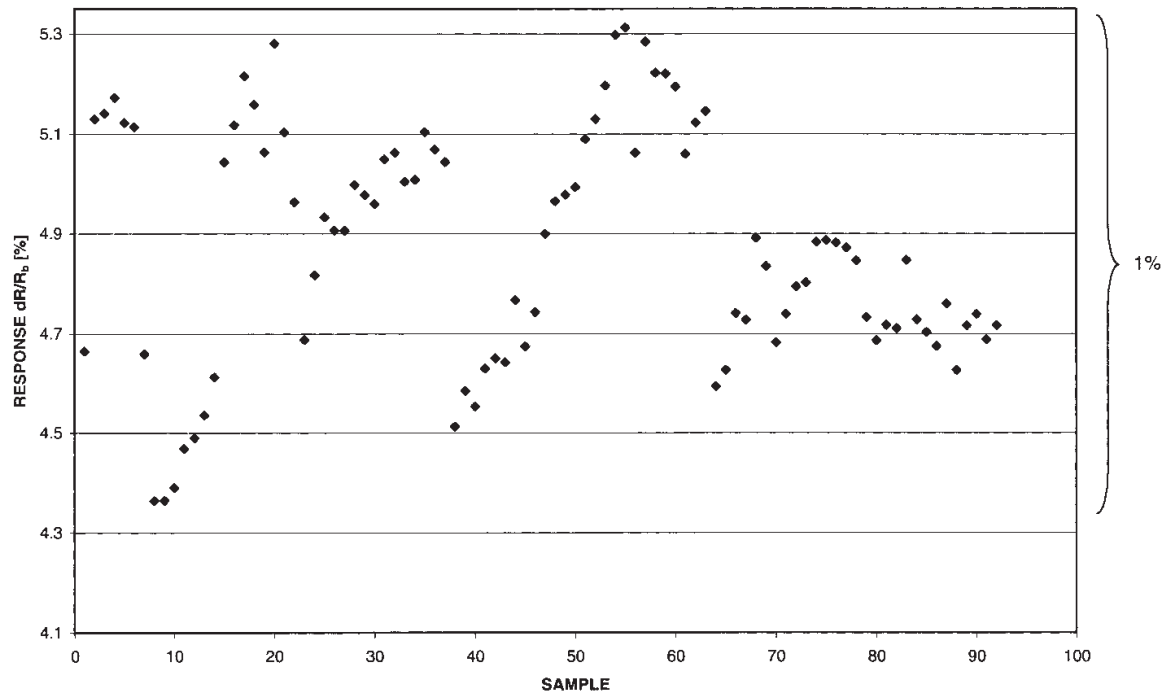

a)

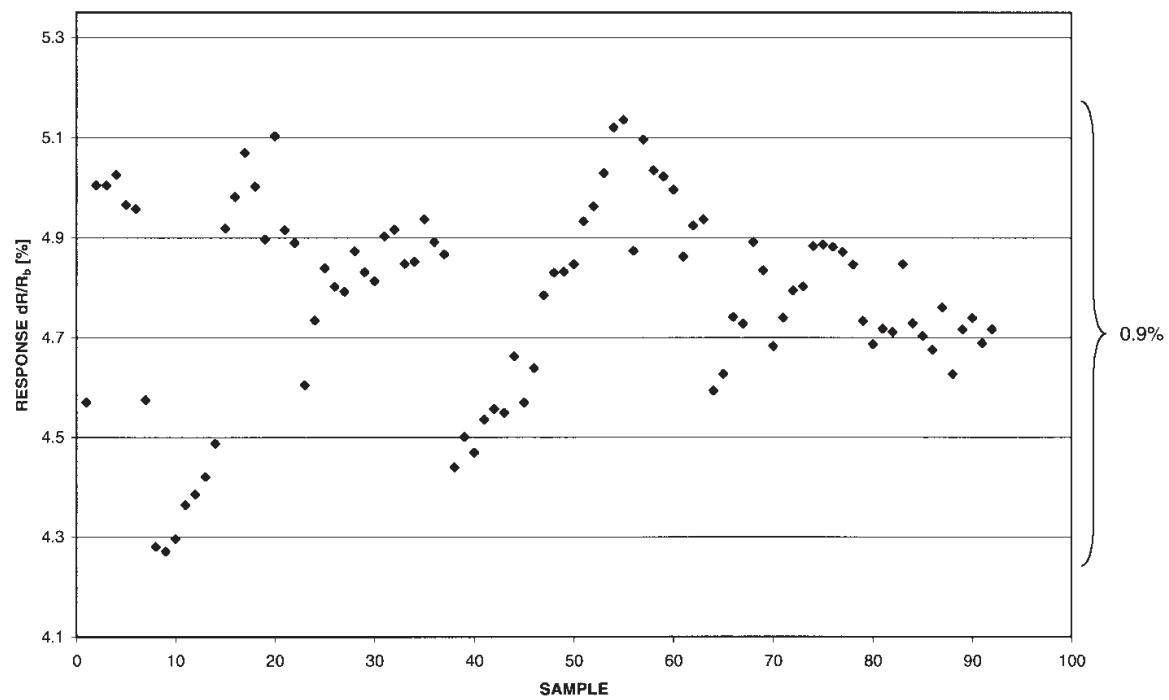

b)

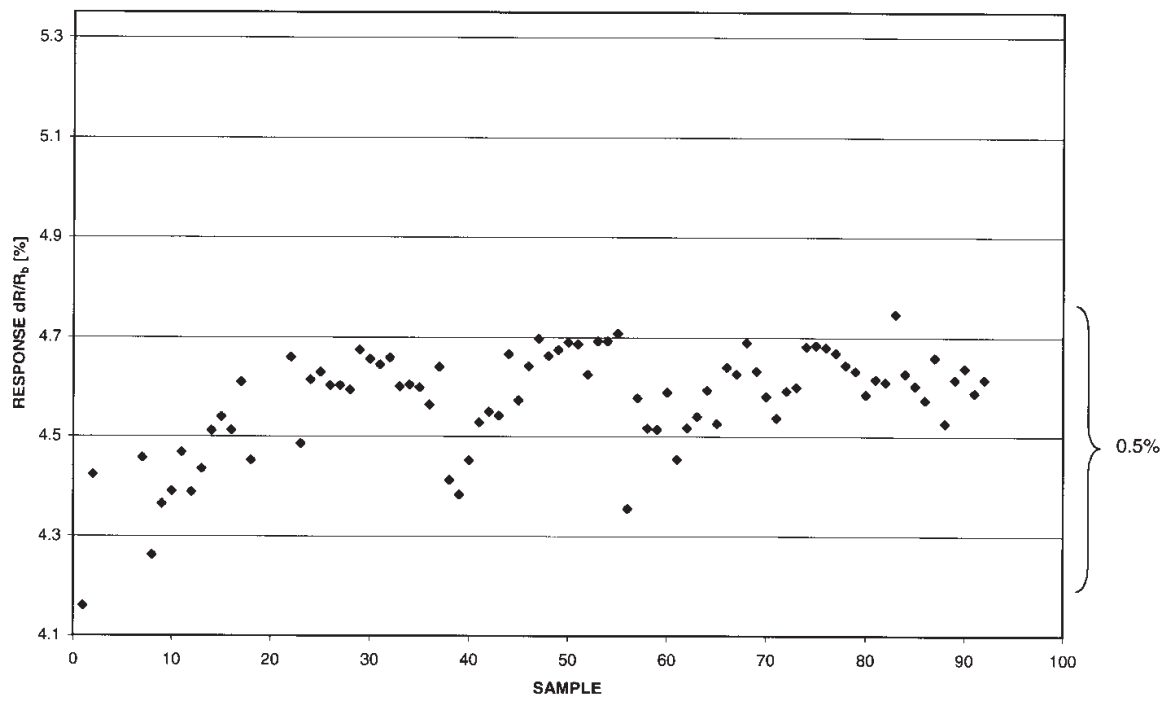

Fig. 8 Comparison of uncorrected and corrected measurements

Caption: a) Maximum response $\left(\mathrm{dR} / \mathrm{R}_{\mathrm{b}}\right)$ of sensor 458 without correction

b) Maximum response $\left(\mathrm{dR} / \mathrm{R}_{\mathrm{b}}\right)$ of sensor 458 with a linear parametric correction to compensate for the drift observed from one hour to the next. The data for the correction were taken

C) between $7 \mathrm{am}$ and $8 \mathrm{pm}$.

c) Maximum response $\left(d R / R_{b}\right)$ of sensor 458 with a linear parametric correction to compensate for the instability due to the temperature variation of the sensor module. The data for the correction were taken between 30.3 and $31.0{ }^{\circ} \mathrm{C}$. 
suitable for products having a constant relative humidity as well as presenting well-differentiated responses so that the residual response variation could be neglected. Only a few rare products correspond to the above-mentioned criteria. If the technical control as well as the understanding of the working mechanism of $\mathrm{CP}$ sensors are not improved, the future of this technology as sensor for volatile compounds is seriously jeopardised.

\section{Acknowledgement}

The authors would like to thank Mrs. Gerda Urbach, Food Science Australia, Melbourne Laboratory, for her careful review and correction of the manuscript.

\section{References}

1. Schaller, E.; Bosset, J.O.; Escher, F. Lebensm.-Wiss. $u$. Technol. 1998, 31, 305-316.

2. Shiers, V.P. In Food Ingredients Europe: Conference Proceedings; Maarssen Ed.; Miller Freeman Technical: Frankfurt, 1995; pp 198-200.

3. Mielle, P. Trends in Food Science \& Technology, Special Issue on Flavour Perception 1996, 7, 432-438.

4. Amrani, M.E.H.; Persaud, K.C.; Payne, P.A. Meas. Sci. Technol. 1995, 6, 1500-1507.

5. Hodgins, D.; Simmonds, D. J. Automatic Chem. 1995, 17(5), 179-185.

6. Hodgins, D. In Techniques for analyzing food aroma; Marsili, R., Ed.; Marcel Dekker Inc:New York, 1997; pp 331-371.

7. Wünsche, L.F.; Vuilleumier, C.; Keller, U.; Byfield, M.P.; May, I.P.; Kearney, M.J. Proceedings of the 13th International Congress of Flavours, Fragrances and Essential Oils, 15-19 October 1995, Istanbul, Turkey, 1995, 3, 295-313.
8. Slater, J.M.; Watt, E.J.; Neville, J.; Fiain, P.M.; Donald, W.J. Analyst 1992, 117, 1265-1270.

9. Yueqiang, S.; Carneiro, K.; Ping, W.; Renyuan, Q. In Electronic properties of conjugated polymers; Kuzmany, H., Mehring, M., Roth, S., Eds.; Spinger Series in Solid-State Sciences; Spinger-Verlag: Berlin, vol. 76, 1987; pp 31-35.

10. Yueqiang, S.; Carneiro, K.; Jacobsen, C.; Renyuan, Q.; Qiu, J. Synthetic Metals 1987, 18, 77-83.

11. Persaud, K.C.; Qutob, A.A.; Travers, P.; Pisanelli, A.M.; Szyszko, S. Proceedings of the 11th Symposium on Olfaction and Taste and of the 27th Japanese Symposium on Taste and Smell, Tokyo: Springer-Verlag 1994, pp 708-710.

12. Anklam, E.; Lipp, M.; Radovic, B.; Chiavaro, E.; Palla, G. Food chemistry 1998, 61(1-2), 243-248.

13. Bartlett, P.N.; Ling-Chung, S.K. Sensors and Actuators 1989, 19, 141-150.

14. Meijerink, M.G.H. Ph. D. Thesis, Institute of Microtechnology, University of Neuchâtel, Mai 1999.

15. Persaud, K.C.; Travers, P.J. In Handbook of Biosensors and Electronic Noses; Kress-Rogers, E., Ed.; Frankfurt: CRC Press 1997; pp 563-592.

16. Bartlett, P.N.; Archer, P.B.M.; Ling-Chung, S.K. Sensors and Actuators 1989, 19, 125-140.

17. Pearce, T.C.; Gardner, J.W.; Friel, S.; Bartlett, P.N.; Blair, N. Analyst 1993, 118, 371-377.

18. Gardner, J.W.; Bartlett, P.N. Transducers '95 Eurosensors IX, The 8th International Conference on Solid-State Sensors and Actuators and Eurosensors IX, Stockholm, Sweden (June 2529), 1995, pp 671-686.

19. Slater, J.M.; Paynter, J.; Watt, E.J. Analyst 1993, 118, 379-384.

20. Gretsch, C.; Delarue, J.; Toury, A.; Visani, P.; Liardon, R. ASIC 17e Colloque, Nairobi, 1997, 183-190.

21. Miasik, J.J.; Hooper, A.; Tofield, B.C. J. Chem. Soc. Faraday Trans. 1986, 1(82), 1117-1126.

22. Neaves, P.I.; Hatfield, J.V. Sensors and Actuators B 1995, 2627, 223-231.

23. Pearce, T.C.; Gardner, J.W. Analyst 1998, 123(10), 2057-2066. 